CURRENT TOPIC

\title{
Attention deficit and hyperactivity disorder, methylphenidate, and epilepsy
}

\author{
M Tan, R Appleton
}

Arch Dis Child 2005;90:57-59. doi: 10.1136/adc.2003.048504

Attention deficit hyperactivity disorder (ADHD) is characterised by inattention, impulsivity, and hyperactivity. The DSM-IV diagnosis of ADHD requires the presence of six of nine items or features that must have been present for at least six months, to have had an onset before 7 years of age, and to have resulted in significant distress or impairment. ' In the general population, the prevalence of ADHD is approximately $5 \% .^{2}$ There is a high co-morbidity of epilepsy and attentional and behavioural problems, ${ }^{34}$ including $A D H D$, and it has been estimated that at least $20 \%$ of patients with epilepsy may present with features of ADHD. ${ }^{5}$

\footnotetext{
Correspondence to Dr R Appleton, The Roald Dahl EEG Unit, Department of Neurology, Royal Liverpool Children's Hospital (Alder Hey), Eaton Road, Liverpool L12 2AP, UK; Richard. Appleton@RLCH-TR. NWEST.NHS.UK
}

Accepted 25 April 2004
M ethylphenidate, a psychostimulant and derivative of amphetamine, is a controlled drug that is recommended for use as part of a treatment programme for children with a confirmed diagnosis of attention deficit hyperactivity disorder (ADHD). ${ }^{67}$ The drug is reported to be effective in up to three quarters of children with $\mathrm{ADHD}^{8}$ and is generally associated with few side effects. However, there is a belief that methylphenidate may lower seizure threshold and consequently concerns have been raised regarding the use of the drug in children who have ADHD and epilepsy. This predominantly reflects the authors' personal experience from discussions with colleagues in community and child and adolescent mental health but also reflects statements within commonly used prescribing manuals, including Medicines for Children (published by the Royal College of Paediatrics and Child Health, 2003, pp. 403-4). Both the British National Formulary (BNF, September 2003, pp. 197-8) and the Monthly Index of Medical Specialities (MIMS, October 2003, pp. 122-3) recommend caution when prescribing psychostimulants, including dexamphetamine and methylphenidate, to patients with epilepsy, and discontinuation of these drugs if seizures occur. Given the relatively common co-occurrence of epilepsy and ADHD, this is a potentially important issue.

The aim of this article is to briefly review the relation between ADHD and epilepsy, and to address the use of methylphenidate in children with both epilepsy and ADHD. It is not within the remit or scope of this article to discuss either the differentiation of ADHD from conditions such as oppositional defiant disorder and conduct disorder or the complex relation between cognitive (including attentional) and behavioural problems and epilepsy. Comprehensive reviews of this relation can be found in recent publications. $^{23}$ Clearly, implicit within this review is the assumption that the diagnosis of ADHD is both appropriate and accurate.

\section{ADHD AND EPILEPSY}

Many hypotheses have been put forward in an attempt to explain the co-existence of or apparent association between ADHD and epilepsy. Attention deficit hyperactivity disorder and epilepsy may exist within a common syndrome complex; ${ }^{9}$ both may have a genetic predisposition; and finally, antiepileptic drugs (AEDs) may contribute to both attentional difficulties and increased levels of activity, including irritability in a number of children. The observation that behavioural and attentional problems may be seen prior to the onset of epilepsy ${ }^{10}$ would suggest that they are unlikely to be the direct effect of clinical seizures or AEDs. A recent study of 51 schoolchildren showed no deficits in attention in children with newly diagnosed idiopathic or cryptogenic epilepsy. ${ }^{11}$ Most studies seem to suggest that the majority of AEDs (other than possibly phenobarbitone and the benzodiazepines) have little effect on cognitive functioning, including attention..$^{22-14}$

The type of epilepsy may also be important, although the evidence is conflicting. Children with complex partial seizures are reported to have significant difficulty with aspects of sustained attention, irrespective of whether there is a preceding or concurrent diagnosis of ADHD. ${ }^{15}$ Although children with ADHD and those with complex partial seizures have similar problems with attention, the mechanism responsible may be different. ${ }^{3}$ Impaired attention has also been described in children with benign childhood epilepsy with centro-temporal (rolandic) spikes (BCECTS). Right sided interictal epileptiform activity in these children interferes with right hemispheric activity including attention. ${ }^{16}$ Other studies have suggested that the seizure or epilepsy type is not important. Williams et al found a statistically significant reduction in visual and verbal attention in 79 children with active and treated epilepsy that was irrespective of seizure type, suggesting a possible general developmental effect of epilepsy or its treatment, or both. ${ }^{17}$ More recently Dunn and colleagues ${ }^{2}$ found that 64 of 175 (36\%) children with

Abbreviations: $A D H D$, attention deficit hyperactivity disorder; AED, antiepileptic activity; CAE, childhood onset absence epilepsy; EEG, electroencephalogram 
epilepsy met DSM-IV criteria for ADHD and that although neither seizure type nor focus of electroencephalographic (EEG) discharge predicted the occurrence of ADHD, there was a tendency for ADHD to occur more frequently in those with generalised seizures.

Clearly, children with childhood onset absence epilepsy (CAE) may present with poor attention or concentration because of frequent seizures. It has been our experience (and one that is likely to be shared by others) that a number of children with CAE have been wrongly diagnosed as having ADHD. However, the diagnosis of CAE should be obvious from the history, including (typically) being able to induce an absence seizure during hyperventilation undertaken in the outpatient clinic. In addition, and in contrast to ADHD, most children with CAE are girls and impulsivity/hyperactivity rarely complicates this specific epilepsy syndrome.

Electroencephalography has also been reported to be abnormal in children with ADHD, some showing epileptiform activity. The reported incidence ranges from $6.1 \%$ of 476 of children with ADHD (compared to $3.5 \%$ of 3726, normal, school age children), ${ }^{18}$ to $30.1 \%$ of 176 children with ADHD. ${ }^{19}$ In this latter study, EEG recordings showed predominantly focal rather than generalised spike and wave complexes. ${ }^{19}$ Holtman et al reported that the frequency of centro-temporal (rolandic) spikes in children with ADHD was significantly higher than expected from normal children, based on epidemiological studies (5.6\% versus $2.4 \%$ respectively), ${ }^{20}$ and have therefore suggested that all children being evaluated for a possible diagnosis of ADHD should undergo EEG, irrespective of whether have they have experienced any epileptic seizures. ${ }^{21}$ The authors make no comment on whether and how they would further investigate and treat children with rolandic spikes and ADHD.

Finally, although children with EEG abnormalities who do not have epilepsy are assumed to be at risk of developing seizures, there are insufficient data to support this hypothesis. ${ }^{22}$

\section{IS METHYLPHENIDATE EFFECTIVE AND SAFE IN CHILDREN WITH ADHD AND EPILEPSY?}

There is little or no published data to suggest that psychostimulants, including methylphenidate, contribute to the de novo development of epilepsy or exacerbate seizures in children with an established diagnosis of epilepsy. Such an association would be unexpected for a number of reasons. Firstly, a new development of epilepsy or an exacerbation of pre-existing epilepsy and the use of methylphenidate may be entirely coincidental, reflecting the natural history of the epilepsy in any individual. Secondly, methylphenidate may interfere with sleep and as a consequence, the drug may lower the threshold for epileptic seizures resulting in deterioration in seizure control. Thirdly, based on what is still a limited understanding of why an epileptic seizure starts (and stops) spontaneously, there is no reason to believe that methylphenidate should be pro-convulsant and therefore lower seizure threshold. The drug primarily acts on the presynaptic reuptake of the neurotransmitters noradrenaline and dopamine rather than the neurotransmitters commonly associated with the pathophysiology of seizures (gamma aminobutyric acid (GABA), glutamic and aspartic acids). It is not known to affect calcium or sodium channels, which are also implicated in the pathophysiology of many of the epilepsies. Finally, although methylphenidate may inhibit the metabolism of phenobarbitone, primidone, phenytoin, and ethosuximide (ETX), this is unlikely to have any significant clinical impact as these drugs are only very rarely (or occasionally in the case of ETX), used in the long term treatment of epilepsy in children. There is little or no information on whether it interacts significantly with the newer antiepileptic drugs. None of these theoretical mechanisms represent a direct effect of methylphenidate on epilepsy.

There is considerable anecdotal evidence to suggest that methylphenidate is safe in children whose seizures are well controlled with antiepileptic drugs. Feldman et al, in evaluating 10 patients with epilepsy who were seizure-free and who also had ADHD, found that methylphenidate was effective in treating the ADHD symptoms in the majority, and none of the patients experienced seizures during the 10 week study period. ${ }^{23}$ All patients had abnormal baseline EEGs that did not alter significantly during the study period. ${ }^{23}$ GrossTsur et al studied 30 children who had active seizures before starting methylphenidate. The 25 children who were seizurefree on antiepileptic medication remained seizure-free ${ }^{5}$ and the remaining five who had ongoing seizures showed no significant increase in seizure frequency during the four month study.

Gucuyener et al examined the safety and efficacy of methylphenidate in the management of two groups of patients with ADHD: one with epilepsy and one without epilepsy but with EEG abnormalities. ${ }^{6}$ One hundred and nineteen children were followed up for 12 months. Methylphenidate was effective in controlling ADHD symptomatology in both groups. No seizures were observed in those with ADHD and epileptiform abnormalities on EEG. There was also no significant change in mean seizure frequency in the group with active epilepsy. In addition, the EEG improved, including becoming normal, in many patients after receiving methylphenidate. This finding is consistent with that of an earlier study reporting EEG findings in children before and six months after commencing stimulant therapy. ${ }^{24}$

In contrast, Hemmer and colleagues ${ }^{25}$ reported different findings in their study of 234 children with ADHD but without epilepsy who underwent EEG examination prior to starting methylphenidate. Following the introduction of methylphenidate, three of the 30 children with an epileptiform EEG developed seizures. The incidence in those with centro-temporal (rolandic) spikes was higher at $16.7 \%$. The incidence of seizures was only $0.6 \%$ in the group who did not have epileptiform EEGs. Although the authors concluded that epileptiform EEGs in neurologically normal children with ADHD could predict the "eventual occurrence of seizure", this risk cannot (and should not) necessarily be attributed to the use of the drug. ${ }^{25}$

Other retrospective data published in abstract form ${ }^{2627}$ have also reported the beneficial use of methylphenidate in those with ADHD and epilepsy. Wroblewski and colleagues ${ }^{26}$ reported a trend towards a lower incidence of seizures in those patients with traumatic brain injury and epilepsy. McBride and colleagues ${ }^{27}$ found that none of their 23 children with a prior history of seizures $(n=20)$ or epileptiform abnormalities on EEG $(n=3)$ and who were treated with methylphenidate, showed any seizure exacerbation or developed new seizures for up to four years after receiving the drug.

In summary, most studies have shown that methylphenidate improves symptoms of ADHD and enhances the performance in children with both ADHD and epilepsy. It does not appear to exacerbate seizures in children with either well controlled or active epilepsy. There is conflicting evidence as to whether seizures occur more frequently in those without epilepsy but who have abnormal EEG abnormalities. The presence or absence of rolandic spikes on the EEG in children with a diagnosis of ADHD are of uncertain clinical significance and should not, in isolation, be used to refute a clinical diagnosis of ADHD. It is also important to emphasise that the accurate interpretation of any EEG depends on obtaining a successful recording that is as 
artefact-free as possible (and that any artefacts are correctly identified) and knowledge of both the normal maturational development of the EEG during early childhood as well as the recognition of genuine abnormalities. This is often difficult in view of the age and behavioural characteristics of this specific population and potentially limited expertise of those undertaking and reporting EEGs in young children. Finally, the definition of "epileptiform" activity on EEG varies between studies. These practical and experiential difficulties may in part explain the reported different EEG findings in children with ADHD. ${ }^{2425} 27$ This emphasises the importance of diagnosing ADHD on the basis of clinical criteria and by medical and psychological staff who are experienced in the diagnosis and management of childhood behavioural disorders.

Clearly, the literature appraised and evidence cited in this brief review are largely anecdotal and not based on randomised clinical trial data. In addition, any conclusions or recommendations will obviously be dependent on accurate and consistent diagnoses of both ADHD and epilepsy.

\section{CONCLUSION}

There is no published evidence to suggest that either the short or long term treatment with methylphenidate increases the risk of developing seizures in children with ADHD. Studies to date have also suggested that methylphenidate would appear to be safe to use in children who have active or well controlled epilepsy. There is little or no evidence indicating that the drug interacts significantly with the more commonly prescribed antiepileptic drugs. Finally, there is insufficient evidence to justify undertaking an EEG in all children with an obvious clinical diagnosis of ADHD and without epilepsy prior to administration of methylphenidate. Where an EEG is inappropriately undertaken without a clinical diagnosis of epilepsy, any abnormalities identified on the EEG must be interpreted appropriately and should not influence the treatment of ADHD.

Although there is no identified contraindication to the use of methylphenidate in children with pre-existing epilepsy, it would clearly be important to monitor seizure frequency in the first few weeks and months after prescribing this stimulant drug.

\section{REFERENCES}

1 American Psychiatric Association. Diagnostic and statistical manual of mental disorders, 4th edn. Washington, DC: APA Press, 1994.

2 Dunn DW, Austin JK, Harezlak J, et al. ADHD and epilepsy in childhood. Dev Med Child Neurol 2003;45:50-4.
3 Sanchez-Carpentiro R, Neville B. Attentional ability in children with epilepsy. Epilepsia 2003;44:1340-9.

4 Gucuyener K, Erdemoglu K, Senol S, et al. Use of methylphenidate for attention-deficit hyperactivity disorder in patients with epilepsy or electroencephalographic abnormalities. J Child Neurol 2003;18:109-12.

5 Gross-Tsur V, Manor O, van der Meere J, et al. Epilepsy and attention deficit hyperactivity disorder: is methylphenidate safe and effective. J Pediatr 1997; 130:670-4

6 National Institute for Clinical Excellence (NICE). Guidance on the use of methylphenidate (Ritalin, Equasym) for ADHD in childhood, NICE guidance no.13. London: National Institute for Clinical Excellence, 2000

7 Scottish Intercollegiate Guidelines Network (SIGN). Attention deficit and hyperkinetic disorders in children and young people, SIGN publication no.52. Edinburgh: SIGN, 2001

8 Stein MA, Blondis TA, Schnitzler ER, et al. Methylphenidate dosing: twice daily versus three times daily. Pediatrics 1996;98:748-56.

9 Weinberg WA, Harper CR, Schraufnagel CD, et al. Attention deficit hyperactivity disorder: a disease or a symptom complex. J Pediatr 1997; 130:665-9.

10 Austin JK, Harezlak J, Dunn DW, et al. Behaviour problems in children before first recognised seizure. Pediatrics 2001;107:115-22.

11 Oostrom KJ, Schouten A, Kruitwagen CL, et al. Attention deficits are not characteristic of schoolchildren with newly diagnosed idiopathic or cryptogenic epilepsy. Epilepsia 2002;43:301-10.

12 Aldenkamp AP, Alpherts WCJ, Blennow G, et al. Withdrawal of antiepileptic medication in children-effects on cognitive function: the Multicenter Holmfrid Study. Neurology 1993;43:41-50.

13 Bourgeois BFD. Antiepileptic drugs, learning and behavior in childhood epilepsy. Epilepsia 1998;39:913-21.

14 Williams J, Bates, S, Griebel ML, et al. Does short-term antiepileptic drug treatment in children result in cognitive or behavioral changes. Epilepsia 1998:39:1064-9

15 Semrud-Clikeman M, Wical B. Components of attention in children with complex partial seizures with and without ADHD. Epilepsia 1999;40:211-15.

16 Piccirilli M, D'Alessandro P, Sciarma T, et al. Attentional problems in epilepsy: possible significance of epileptogenic focus. Epilepsia 1994;35:1091-6.

17 Williams J, Griebel ML, Dykman RA. Neuropsychological patterns in pediatric epilepsy. Seizure 1998;7:223-8.

18 Richer LP, Shevell MI, Rosenblatt BR. Epileptiform abnormalities in children with attention-deficit-hyperactivity disorder. Pediatr Neurol 2002;26:125-9.

19 Hughes JR, Deleo AJ, Melyn MA. The electroencephalogram in attention deficit-hyperactivity disorder: emphasis on epileptiform discharges. Epilepsy Behav 2000;1:271-7.

20 Holtmann M, Becker K, Kentner-Figura B, et al. Increased frequency of rolandic spikes in ADHD children. Epilepsia 2003;44:1241-4.

21 Becker K, Sinzig JK, Holtmann M. Attention deficits and subclinical epileptiform discharges: are EEG diagnostics in ADHD optional or essential? Dev Med Child Neurol 2004;46:431-2.

22 Anderson KV, Trojaborg W. Risk of development of epileptic seizures in children with subclinical "epileptogenic" EEG abnormalities. Egeskr Laeger 1993; 155:1285-8.

23 Feldman $\mathrm{H}$, Crumrine $\mathrm{P}$, Handen $\mathrm{BL}$, et al. Methylphenidate in children with seizures and attention deficit disorder. Am J Dis Child 1989;143:1081-6.

24 Clarke AR, Barry RJ, Bond D, et al. Effects of stimulant medications on the EEG of children with attention-deficit/hyperactivity disorder. Psychopharmacology 2002;164:277-84.

25 Hemmer SA, Pasternak JF, Zecker SG, et al. Stimulant therapy and seizure risk in children with ADHD. Pediatr Neurol 2001;24:99-102.

26 Wroblewski BA, Leary JM, Phelan AM, et al. Methylphenidate and seizure frequency in brain-injured patients with seizure disorders. J Clin Psychiatry 1992:53:86-9.

27 McBride MC, Wang DD, Torres CF. Methylphenidate in therapeutic doses does not lower seizure threshold [abstract]. Ann Neurol 1986;20:428. 